\title{
Atomic Column Elemental Mapping by STEM-Moiré Method
}

\author{
Eiji Okunishi ${ }^{1}$, Noriaki Endo ${ }^{1}$ and Yukihito Kondo ${ }^{1}$ \\ ${ }^{1 .}$ EM Business Unit, JEOL Ltd., 3-1-2 Musashino Akishima, Tokyo 196-8558, Japan.
}

Atomic column elemental mapping is realized by electron energy loss spectroscopy (EELS) and/or energy dispersive X-ray spectroscopy (EDS) [1], with modern technologies of electron microscopy such as an aberration corrector [2]. The method is widely utilized in materials and physical sciences, since atomic species and positions in a crystalline specimen can be determined directly. A crucial issue to perform the mapping is specimen damage due to high electron dose onto a specimen, since ionization cross section for the core electron excitation is so low.

STEM-moiré method is used for a strain measurement [3]. The accuracy reaches less than $0.2 \%$. And the method is used to measure strain in a semiconductor device [4]. The STEM moiré fringe for a regularly periodic lattice arises when the interval of pixels is close to a lattice spacing of crystalline or multiple of it [3]. With the proper pixel intervals in $\mathrm{x}$ and $\mathrm{y}$ directions, the moiré fringe shows the 2D magnified moiré lattice, due to under-sampling effect, as illustrated in Fig. 1(a) and 1(b) [5]. This paper reports a method to perform the atomic column elemental mapping with less electron dose on the specimen, better signal-to-noise ratio and higher pixel resolution, utilizing the STEM-moiré method.

Spacing for translational STEM moiré fringes $\left(d_{m}\right)$ is geometrically determined as $d_{m}=d_{R} r /|1-\mathrm{r}|$, where $d_{R}$ and $r$ are a pixel interval and the ratio of the pixel interval $\left(d_{R}=d_{L} r\right)$ to a lattice spacing $\left(d_{L}\right)$. The magnification $(M)$ of moiré lattice to the original lattice is determined as $M=d_{m} / d_{L}$ or $M=|1-r|^{-1}$. A magnified moiré lattice is formed with signals picked from many original lattices, resulting in reduced electron dose by $M^{-2}$ on the specimen to form an atomic column image with an equal pixel resolution. The 2D magnified moiré lattice shows an isotropic magnification to the original lattice in $\mathrm{x}$ and $\mathrm{y}$ directions, if the original lattice is square and $r$ is equal in $\mathrm{x}$ and $\mathrm{y}$ directions.

The specimen for our experiment was chosen to be $\mathrm{SrTiO}_{3}$ [001] that has a square lattice. The microscope used in our experiment was an aberration corrected microscope (JEM-ARM200F), equipped with a cold FEG, a silicon drift type EDS and an EELS. Figs. 2(a)-2(c) show the high angle annular dark field (HAADF) images of magnified moiré lattice at various $\mathrm{r}$. The magnification (M) increases as $r$ approaches to one ( $r=$ 1.29, 1.11, 1.04, M = 3.44, 9.16, 25.0). Figs. 2(d)-2(o) show simultaneously obtained elemental maps of $\mathrm{Sr}$, Ti and O, detected with the EDS. Each element was clearly separated on each magnified moiré elemental map. And it has to be said that no beam damage on the specimen was observed after the mapping. Figs. 3(a) and 3(b) show the oxygen map of magnified moiré lattice at $r=1.04$ and its line profiles which are plotted along the dotted rectangles shown in Fig. 3(a). The profiles clearly show the peaks at oxygen sites. In contrast to that, the less magnified map or non-magnified map (not shown here) showed lower pixel resolution, resulting in non-recognition of the oxygen peaks. It can be said that the atomic column map with high pixel resolution is obtainable by this magnified moiré mapping method.

In conclusion, the STEM moiré method was successfully applied to atomic column elemental mapping. The method can be applicable to measure detailed physical properties such as delocalization or 
channeling in the crystalline specimens as well as the precise atomic column map with higher pixel resolution, better signal-to-noise ratio and less electron dose than the direct atomic column mapping.

References:

[1] K Kimoto et al., Nature 450 (2007), p. 702.

[2] E Okunishi et al., Microsc. Microanal. 12 (Suppl. 2) (2006), p. 1150.

[3] N Endo and Y Kondo, Microsc. Microanal. 19 (Suppl. 2) (2013), p. 346.

[4] S Kim et al., Appl. Phys. Lett. 102 (2013), p. 161604.

[5] D Su and Y Zhu, Ultramicroscopy 1103 (2010), p. 229.

(a)

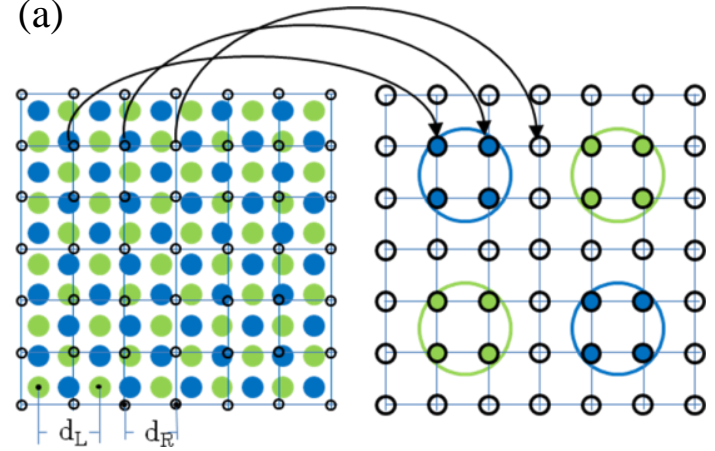

(b)

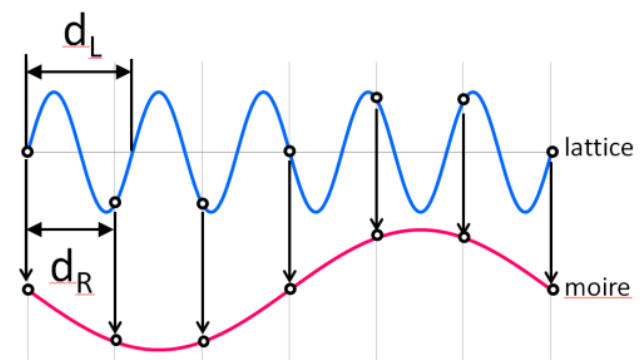

Figure 1. (a) A scheme explaining magnified moiré lattice. The intensity of a pixel in magnified lattice (right panel in (a)) is picked from original lattice (left panel in (a)). The intensity of the next pixel in magnified lattice is picked from the slightly shifted position from the next lattice point by dL-dR. Consequently, with a series of the picking, magnified lattice is formed. (b) The principle of undersampling or moiré fringe, where $\mathrm{d}_{\mathrm{R}}$ and $\mathrm{d}_{\mathrm{L}}$ are pixel interval and lattice spacing.

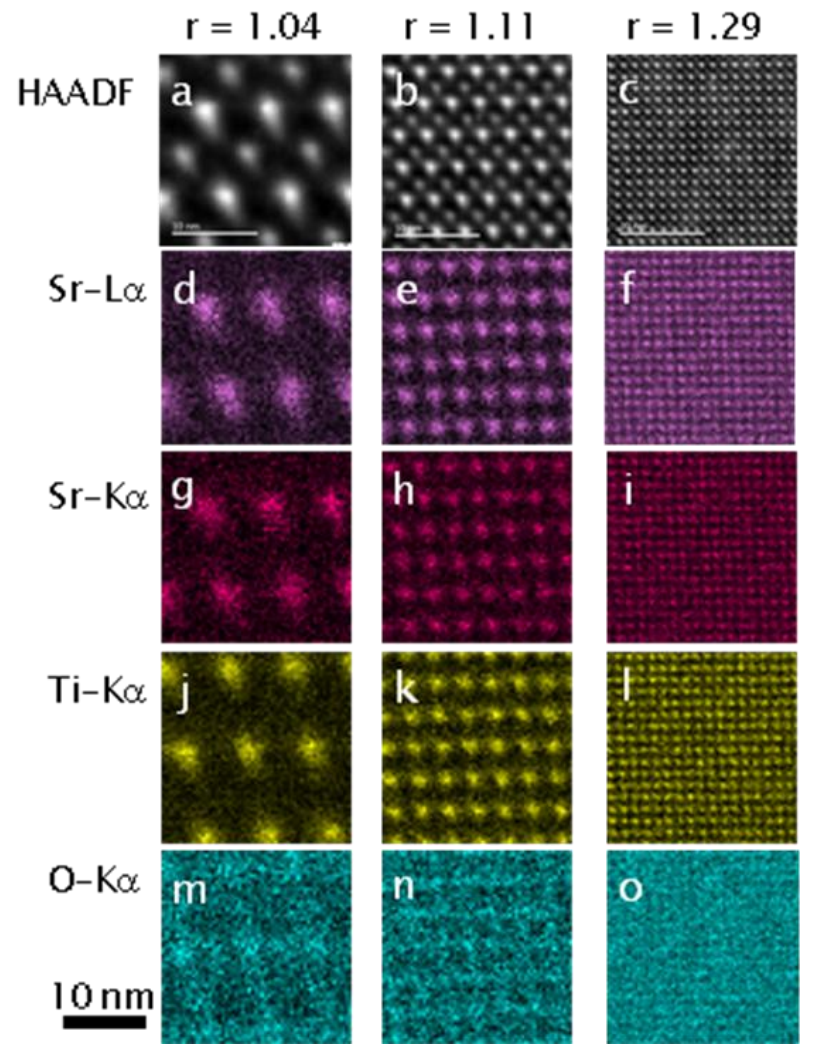

Figure 2. (a)-(d) show HAADF images of magnified moiré lattice of $\mathrm{SrTiO}_{3}$ [001] at various r. The magnification increases as $r$ approaches to one. (d)-(o) show simultaneously obtained elemental maps of Sr-La, Sr-Ka, Ti-Ka and O-Ka. Slight distortions in the magnified lattices are due to imperfections of orthogonality in $\mathrm{x} \& \mathrm{y}$ scanning directions and equality in $\mathrm{x} \& \mathrm{y}$ intervals of a pixel on the specimen.

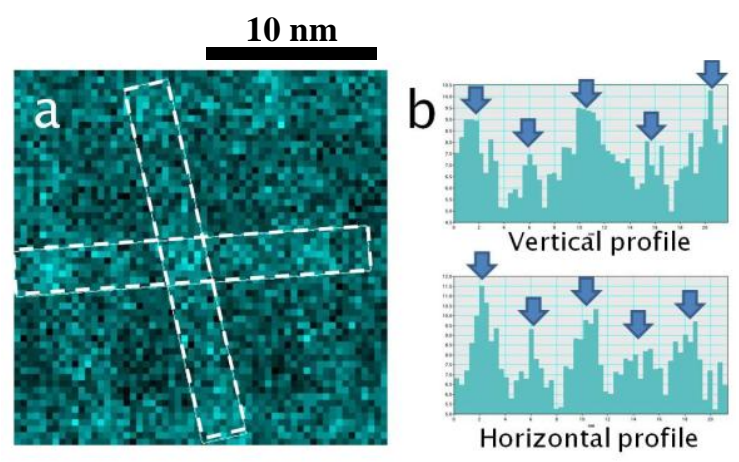

Figure 3. (a) Oxygen map of magnified moiré lattice at $\boldsymbol{r}=1.04$. (b) Line profiles along the dotted rectangles in (a), showing peaks at oxygen sites. 